\title{
Calculation Method of Twist Error of Aero-Engine Blade Based on Three-Coordinate Measuring Data
}

\author{
Chao $\mathrm{LI}^{1, *}$, Zhi HUANG ${ }^{1,2}$ and Yun HUANG ${ }^{2}$ \\ ${ }^{1}$ University of Electronic Science and Technology of China, School of Mechatronics Engineering, \\ 611731 Chengdu, China \\ ${ }^{2}$ Chongqing University, College of Mechanical Engineering, 400044 Chongqing, China \\ *185824133@qq.com
}

Keywords: Aero-Engine, Blade, Twist error, Least squares, Minimum zone.

\begin{abstract}
Blade is one of the core components of the aero-engine, which requires a high degree of geometric dimension precision, so strict geometric shape detection and evaluation is very necessary to guarantee blade machining quality. Twist error is one of the important parameters of blade quality evaluation, which directly affects the evaluation results. In this paper, two kinds of methods are used to calculate the twist error of blade at first, and the calculation method based on the definition of twist degree is studied emphatically. Finally, the twist error extraction experiment was carried out with the actual measurement data of blade, and the validity of the method was verified by comparison.
\end{abstract}

\section{Introduction}

Blade is a kind of typical free-form surface part. Its surface quality directly affects the energy conversion efficiency of the engine, so it must use advanced detection technology to measure and assess its profile. The twist design of blade improves its aerodynamic performance, but it also increases the complexity of detection. Exact calculation of twist error has been one of the difficulties in the quality evaluation of blade.

The calculation method of twist error of blade cross-section can be divided into two categories. The first category method is that the angle difference between the corresponding characteristic line of measured data and theoretical data is approximated as twist error[1,2]. In practical applications, the angle difference between the common tangent of the leading edge and the trailing edge of the blade is usually taken as the twist error. The computational principle of this method is easy to understand, and can quickly get the twist error. The second category is that the objective function should be constructed to compute the rotation angle when the measured section data aligns with the theoretical section line. The method which belongs to the second category is based on the definition of twist degree, and it is more accurate and reliable than the former one. However, due to the complexity of the objective function, and the problem to be solved is a nonlinear programming problem, all the above things make it difficult to solve the exact rotation angle. Therefore, in order to obtain the twist error accurately, the calculation of twist error is studied by using the idea of least squares and minimum zone, and the validity of the calculation method has been proved based on the coordinate measurement data.

\section{The Establishment of Objective Function}

\section{Least Squares Principle (LSP)}

The principle of least squares gives a criterion for data alignment. It seeks the best match for the data by minimizing the square of the error. The measuring data and the CAD model have similar profiles at the same height (the $Z$-axis is the same). In accordance with the principle of least squares, the best alignment effect is achieved when the sum of squares of the distances between the corresponding points on the similar cross-section lines is at a minimum. Assuming that the theoretical point of the blade CAD model on the cross-section is $P_{i}\left(a_{i}, b_{i}, z\right)$, and the corresponding measuring point is $Q_{i}^{f}\left(x_{i}^{f}, y_{i}^{f}, z\right)$. Assuming that matrix $T$ is the transformation matrix of the measuring point. Since the 
transformation has only the rotation around the $Z$ axis and the movement along the $X$ and $Y$ axes, $T$ can be expressed as:

$$
T=\left[\begin{array}{cccc}
\cos y & -\sin y & 0 & L_{x y} \\
\sin y & \cos y & 0 & L_{y} \\
0 & 0 & 1 & 0 \\
0 & 0 & 0 & 1
\end{array}\right]
$$

Where: $\gamma$ is the rotation angle of the measuring data around the $Z$ axis. $L_{x}$ and $L_{y}$ are the moving amounts along the $X$ and $Y$ axes respectively.

Assuming that the coordinate of $Q_{i}^{g}$ after moving and rotating is $Q_{i}\left(x_{i}, y_{i}, z_{i}\right)$. Then the coordinates of $Q_{i}$ can be obtained by cross-multiplying $T$ and $Q_{i}^{t}$ :

$$
\begin{gathered}
Q_{\mathrm{i}}=T \times Q_{\mathrm{i}}^{g} \\
\left\{\begin{array}{c}
x_{\mathrm{i}}=\cos y \cdot x_{\mathrm{i}}^{s}-\sin y \cdot y_{\mathrm{i}}^{g}+L_{x} \\
y_{\mathrm{i}}=\sin y \cdot x_{\mathrm{i}}^{g}+\cos y \cdot y_{\mathrm{i}}^{g}+L_{y} \\
z_{\mathrm{i}}=z
\end{array}\right.
\end{gathered}
$$

By least-squares method, the square of the error should be minimized. Therefore, the following objective function can be established:

$$
\sum_{i=1}^{n}\left[\left(x_{i}-a_{i}\right)^{2}+\left(y_{i}-b_{i}\right)^{2}\right]=\min
$$

Where: $\mathrm{n}$ is the total number of measuring points.

Combining equations (3) and (4), the following objective function equation can be obtained:

$$
\begin{aligned}
& f\left(L_{x}, L_{y}, \gamma\right)=\sum_{i=1}^{n}\left[\left(\cos \gamma \cdot x_{i}^{r}-\sin \gamma \cdot y_{i}^{\prime}+L_{x}-\right.\right. \\
& \left.\left.a_{i}\right)^{2}+\left(\sin \gamma \cdot x_{i}^{r}+\cos \gamma \cdot y_{i}^{\prime}+L_{y}-b_{i}\right)^{2}\right]
\end{aligned}
$$

\section{Minimum Zone Principle (MZP)}

According to the evaluation standard of the minimum area of form deviation of the free curve, When the maximum distance between the measurement point and the theoretical contour goes to its minimum, the measured data and the theoretical curve achieve the best alignment state[3]. In order to minimize the maximum distance, it is necessary to carry out the coordinate's translation transformation and rotation transformation for the measuring point. Assuming that the point $P_{i}\left(x_{i}, y_{i}, z\right)$ is the measurement data of the coordinate measuring machine, $Q_{i}\left(a_{i}, b_{i}, z\right)$ is the corresponding point on the theoretical curve, matrix $T$ is the transformation matrix, matrix $R$ is the rotation matrix, and matrix $M$ is the translation matrix, then matrix $T$ can be expressed as:

$$
T=\left[\begin{array}{cc}
R & M \\
0 & 1
\end{array}\right]=\left[\begin{array}{ccc}
\cos \gamma & -\sin \gamma & L_{x} \\
\sin \gamma & \cos \gamma & L_{y} \\
0 & 0 & 1
\end{array}\right]
$$

Where: $L_{x}$ and $L_{y}$ are translation variables, and $R$ is the rotation variable.

Assuming that $P_{i}^{\prime}\left(x_{i}^{b}, y_{i}^{b}, z_{i}\right)$ is the measured point after the alignment transformation.

$$
P_{i}^{\prime}=T \cdot P_{i}^{T}
$$

The following objective function can be obtained by the definition of the minimum zone principle:

$$
f\left(L_{x,} L_{y s} y\right)=\min \left\{\max \sqrt{\left(x_{i}^{s}-a_{i}\right)^{2}+\left(y_{i}^{t}-b_{i}\right)^{2}}\right\}
$$

Where: $\mathrm{i}=1,2, \cdots, \mathrm{n} . \mathrm{n}$ is the total number of cross-section measurement points.

\section{The Solution of Objective Function}

The key step in solving the objective function is to find the parameters in the transformation matrix.

For the objective function established by least squares principle, the partial derivatives of the three unknown parameters need to be solved respectively, and the result of partial derivative is zero, the rotation angle $\gamma$ of the measured model around the $Z$-axis can be obtained by calculating . The main calculation is as follows: 


$$
\left\{\begin{array}{c}
A\left(L_{y} \cdot \cos \gamma-L_{x} \cdot \sin \gamma\right)+(E+H) \sin \gamma- \\
(F-G) \cos \gamma-B\left(L_{x} \cdot \cos \gamma+L_{y} \cdot \sin \gamma\right)=0 \\
A \cos \gamma-B \sin \gamma+n L_{x}-C=0 \\
A \sin \gamma+B \cos \gamma+n L_{y}-D=0 \\
A=\sum_{i=1}^{n} x_{i}^{g} \\
B=\sum_{i=1}^{n} y_{i}^{b} \\
C=\sum_{i=1}^{n} a_{i} \\
D=\sum_{i=1}^{n} b_{i} \\
E=\sum_{i=1}^{n} a_{i} \cdot x_{i}^{g} \\
F=\sum_{i=1}^{n} b_{i} \cdot x_{i}^{n}, \\
G=\sum_{i=1}^{n} a_{i} \cdot y_{i}^{b} \\
H=\sum_{i=1}^{n} b_{i} \cdot y_{i}^{b} .
\end{array}\right.
$$

What can be obtained from the above equation is that:

$$
\gamma=\operatorname{arctg}\left(\frac{B C-n G+n F-A D}{n E+n H-A C-B D}\right)
$$

For the objective function based on the minimum zone principle, the Davidon-Fletcher-Powell (DFP) algorithm or the genetic algorithm can be used to solve the objective function[4]. In this paper, genetic algorithm is used to solve the objective function. The principle is mainly about generating the new population which contains the potential optimal solutions by operating the operator using selecting, crossing, mutation and other genetic algorithms, and then guiding the optimization calculation by fitness to achieve solution's survival of the fittest. The optimal solution of the problem can be approached step by step, and after all the heuristic search process of the optimal solution is completed, which has strong robustness and global optimization ability. The algorithm design is described as follows:

Step1:Initializing: population size / n: 300; hybridization probability / Pc: 0.9; mutation probability / Pm: 0.01

Step 2: Generating initial population randomly

Step 3: Evaluating the fitness of the population $\mathrm{P}(\mathrm{t})$

Step 4: Population copy $\mathrm{P}^{\prime}(\mathrm{t})$

Step 5: Individual crossover $\mathrm{P}$ " (t)

Step 6: Individual mutation $\mathrm{P}^{\prime \prime}(\mathrm{t})$

Step 7: Evaluating the fitness of the population $\mathrm{P}$ '"(t)

Step 8: Start the next genetic process $\mathrm{P}(\mathrm{t}+1)$

Step 9:Judging the termination condition, if not meet the termination conditions, go to step 3 to continue the process of genetic evolution, otherwise output the current optimal individual, the algorithm ends.

The evaluation fitness function in Step 3 is mainly determined by the value of equation 3. The termination condition in step 9 is mainly determined by the required accuracy of the twist error, and the value of the initialized parameter has a direct impact on the calculation result and the timeconsuming.

\section{Experiment and Analysis}

The experiment is based on the three-coordinate measurement data of certain engine blade, and the calculation method of the twist error is analysed and verified. The blade is measured by contour method, measuring a total of 6 sections. As shown in Fig. 1, the blue point set is the measured data. 


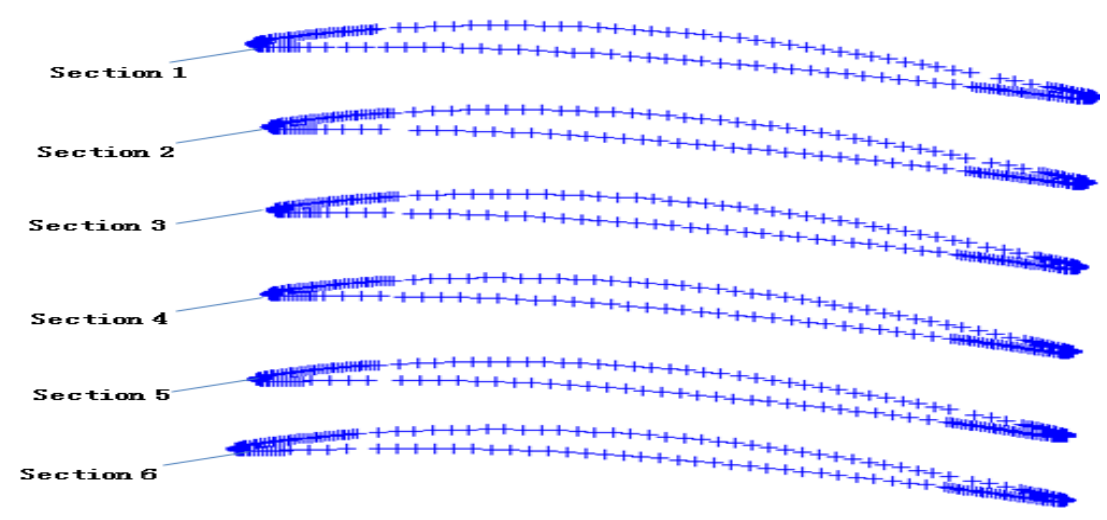

Figure 1. Measuring data of blade.

Figure 2 shows the measuring data and theoretical curves of section 1 . The blue point set is the measured data of section 1 , and the red profile is the theoretical profile.

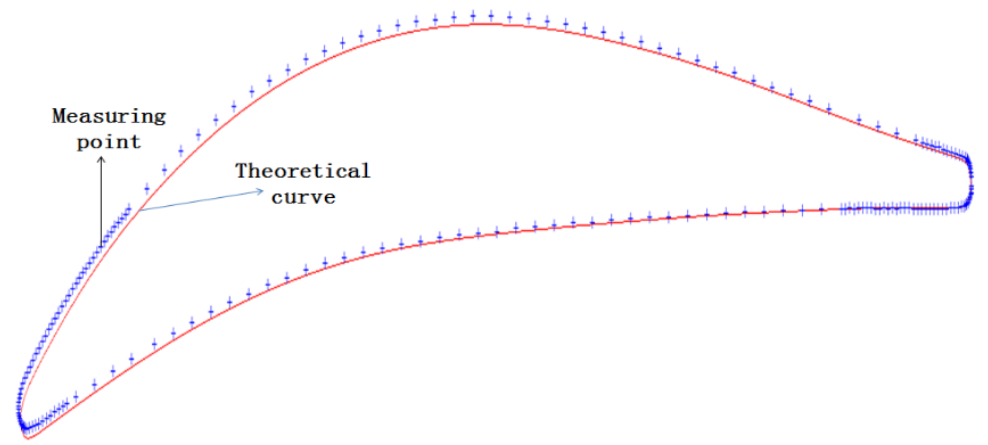

Figure 2. Section 1.

The method which belongs to the first category is used to calculate the twist error, as shown in Fig.3. The area shown in the figure is the leading edge region of the section 1.The blue lines in the figure are the common tangent of the leading and trailing edges, and $\gamma$ is the twist error of this crosssection of blade. This method can obtain the approximate value of the twist error, and the solution of angle difference between the two common tangents is also relatively simple, so this paper does not verify this methods.

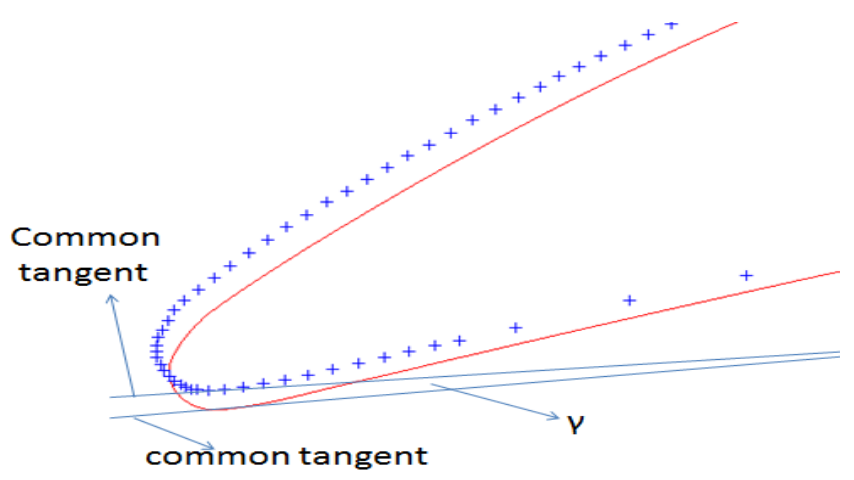

Figure 3. The first category method.

The six sections of the blade are aligned by the second category methods, and the principle of least squares and minimum zone are used to construct the objective function. Taking the section 1 as an example, the position of the measurement point before transformation is shown in Fig.4, and the position of the measurement point after coordinate transformation is shown in Fig. 5. The blue measuring point set in the figure is obtained by the alignment process by the least squares method, and the black measuring point is obtained by the alignment process of the minimum zone method. 


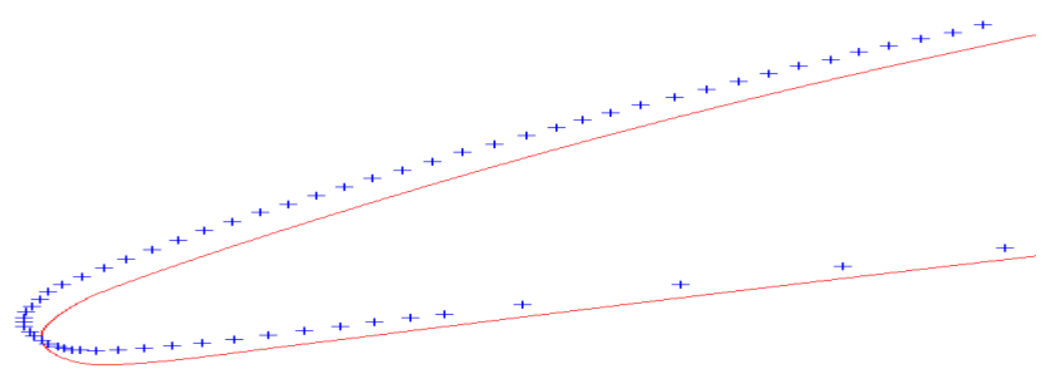

Figure 4. Leading edge of the section 1.

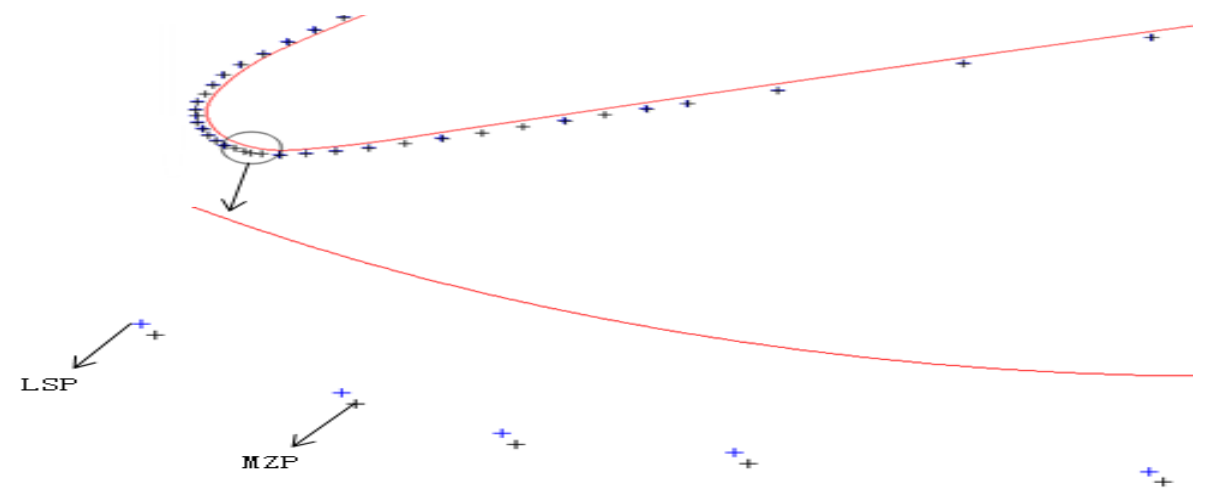

Figure 5. Coordinate transformation.

The parameters of the transformation matrix of each section are obtained by solving the objective function. The values of the each section translation variables $L_{x}$ are shown in Table 1, and the values of the $L_{y}$ are shown in Table 2. The twist error $\gamma$ of each section are shown in Table 3. Table 4 shows the calculation time for these two methods. The value of $L_{x}$ and $L_{y}$ is the position error of the blade, and it is also one of the important parameters of blade quality evaluation.

Table 1 . The values of the $L x$.

\begin{tabular}{|c|c|c|c|}
\hline \multicolumn{3}{|c|}{$L_{x}(\mathrm{~mm})$} \\
\hline Section Method & least squares & Minimum zone & \multirow{2}{*}{ PC-DIMS Blade } \\
\hline 1 & -0.138057 & -0.013067 & -0.012661 \\
\hline 2 & -0.069631 & -0.005361 & -0.003427 \\
\hline 3 & -0.001808 & 0.000886 & 0.000894 \\
\hline 4 & -0.000380 & -0.003646 & 0.000894 \\
\hline 5 & -0.007406 & -0.004747 & -0.004983 \\
\hline 6 & -0.013919 & -0.010407 & -0.010484 \\
\hline
\end{tabular}

Table 2. The values of the $L_{y}$.

\begin{tabular}{|c|c|c|c|}
\hline \multicolumn{4}{|c|}{$L_{y}(\mathrm{~mm})$} \\
\hline $\begin{array}{ll}\text { Section } & \text { Method }\end{array}$ & least squares & Minimum zone & PC-DIMS Blade \\
\hline 1 & 0.062172 & 0.069631 & -0.013919 \\
\hline 2 & 0.073218 & 0.0813866 & 0.079642 \\
\hline 3 & 0.080251 & 0.0871716 & 0.086534 \\
\hline 4 & 0.081651 & 0.0885674 & 0.087924 \\
\hline 5 & 0.084196 & 0.0884985 & 0.088545 \\
\hline 6 & 0.043198 & 0.048521 & 0.048329 \\
\hline
\end{tabular}


Table 3. The twist error of each section of the blade.

\begin{tabular}{|c|c|c|c|}
\hline \multicolumn{4}{|c|}{ Twist Error $\gamma\left({ }^{\circ}\right)$} \\
\hline Section & least squares & Minimum zone & $\begin{array}{c}\text { PC-DIMS } \\
\text { Blade }\end{array}$ \\
\hline 1 & -0.271410 & -0.267342 & -0.267670 \\
\hline 2 & -0.281614 & -0.275113 & -0.275006 \\
\hline 3 & -0.270207 & -0.264978 & -0.265136 \\
\hline 4 & -0.261016 & -0.256426 & -0.256678 \\
\hline 5 & -0.168714 & -0.168922 & -0.168844 \\
\hline 6 & -0.220111 & -0.218604 & -0.218842 \\
\hline
\end{tabular}

Table 4. Computation time.

\begin{tabular}{|c|c|}
\hline Method & Computation Time \\
\hline Least squares & $6264(\mathrm{~ms})$ \\
\hline Minimum zone & $38568(\mathrm{~ms})$ \\
\hline
\end{tabular}

By comparing the above table, it can be concluded that the calculation results obtained by the two methods are very close to the detection results of the PC-DIMS Blade, and the validity of the calculation method of the twist error is proved.

Furthermore, by analyzing Table 4, the comparison between the two methods shows that the least squares method is more efficient than the minimum zone method, and the calculation results of the minimum zone method is closer to the PC-DIMS Blade calculation results.

\section{In Conclusion}

During the process of the extraction of twist error of blade, the second category method should be given priority. The choice of method to establish the objective function should be made by considering the actual situation. The least squares principle is used to meet the requirements of the general accuracy of error evaluation, for the method is more general and the convergence rate is faster. In the case of high error accuracy, the minimum region evaluation principle should be selected for the establishment of the objective function, but this method is usually difficult to solve and the calculation efficiency is low.

\section{Acknowledgements}

This paper is supported by the Fundamental Research Funds for the Central Universities (NO.ZYGX2015J086).

\section{References}

1. Ping Xi, Xiaoxia Sun. Error analysis system of turbine blade based on CAD model[J]. Journal of Beijing University of Aeronautics \& Astronautics, 34.10 (2008): 1159-1162.

2. Zhiguang Peng. Feature Parameters Extraction of the Blade Surface based on the Improved Convex Hull Algorithm[J]. Equipment Manufacturing Technology, 01 37(2012).

3. Ping Liao, Shouyi Yu. Calculating of Flat Complex Curve Form Error Based on Genetic Algorithms with Canonicity Real Number Encoding[J]. Acta Metrologica Sinica, 03 (2003).

4. Chen Z Q. Cross-sectional Feature Parameter, Extraction of Blade Based on Measurement Data[J]. Science Technology \& Engineering, 9, 35(2007). 